\title{
Efetividade de Conversão dos Investimentos em TI na Eficiência dos Bancos Brasileiros
}

\author{
Antonio Carlos Gastaud Maçada \\ João Luiz Becker \\ Guilherme Lerch Lunardi
}

\begin{abstract}
Resumo
Embora estudos acadêmicos revelem pouca relação entre os investimentos em Tecnologia de Informação (TI) e eficiência organizacional, a indústria bancária brasileira tem aumentado significativamente o volume dos seus investimentos em TI visando à implementação de estratégias e na busca de vantagens competitivas. Este artigo procura analisar a eficiência relativa dos investimentos em TI nos bancos brasileiros no período de 1995 a 1999. Propõe-se um modelo para avaliação, utilizando a técnica DEA apoiado no conceito de efetividade de conversão (Weill, 1989). Seguindo método de Pesquisa Operacional, o modelo foi desenvolvido em duas etapas: (1) survey com executivos de bancos para selecionar as variáveis estratégicas organizacionais afetadas pela TI; e (2) entrevistas com executivos para adaptar as variáveis como input e output observáveis em contas de balanço. A maioria (72,2\%) dos bancos pesquisados revela-se eficiente. Os maiores investidores em TI vêm aumentando sua eficiência relativa ao longo do período analisado, ultrapassando os demais. Nem sempre investir em TI é eficiente.
\end{abstract}

Palavras-chave: modelo de eficiência; investimentos em TI; indústria bancária.

\section{Abstract}

Although academic studies have shown a weak connection between investments in Information Technology (IT) and organizational efficiency, the Brazilian bank industry have significantly increased the volume of its investments in IT, aiming at the implementation of strategies and searching competitive advantages. The objective of this paper is to analyze the relative efficiency of IT investments in Brazilian banks from 1995 to 1999. An evaluation model is proposed using the DEA technique, supported on the concept of conversion effectiveness (Weill, 1989). Following the Operations Research methodology, the model was developed in two stages: (1) a survey with banks' executives to select organizational strategic variables affected by IT; and (2) interviews with executives to adapt the variables as input and output observable in balance sheets. The balance sheet data selected in the sample were provided by the Brazilian Central Bank. Most banks (72.2\%) surveyed were found efficient. The biggest IT investors increased their relative efficiency during the period, overtaking the others. On the other hand, investing in IT is not always efficient.

Key words: efficiency model; TI investments; banking industry. 


\section{INTRODUÇÃO}

A Tecnologia de Informação (TI) desempenha papel estratégico significante nas organizações (HENDERSON; VENKATRAMAN, 1999; CROTEAU; BERGERON, 2001). Por esse motivo as organizações são forçadas a investir em TI para se manter competitivas (The Economist, May 2001). Em particular, a indústria bancária tem utilizado a TI como ferramenta estratégica (CHANNON, 1998). Os investimentos em tecnologia dos bancos brasileiros continuam aumentando em percentuais elevados para permitir a ampliação da crescente demanda dos clientes por serviços com maior conforto, segurança e agilidade. Entre 1998 e 2000, verifica-se crescimento significativo da participação nos investimentos dos equipamentos e linhas de comunicação - de 8,4\% para 14,9\% - e dos softwares - de 33\% para 39\% (FEBRABAN, 2001).

Para Bergeron et al. (2001) a relação entre investimentos em TI e eficiência da firma é complexa e multifacetada. Medir a efetividade de conversão dos investimentos em TI, a partir de fatores estratégicos, contribui para reduzir essa complexidade. Weill (1989) desenvolveu o conceito de efetividade de conversão, que é a capacidade para converter as despesas com TI em valores que possam trazer retorno ao investimento da organização. Lucas Jr. (1999) salienta que, se uma organização falhar na implementação e utilização de TI, os resultados da efetividade de conversão serão menores.

A Análise Envoltória de Dados (do inglês, Data Envelopment Analysis - DEA), segundo Troutt et al. (1999), Shafer e Byrd (2000) e Thanassoulis (2001), vem sendo aplicada como alternativa aos modelos econométricos tradicionais no desenvolvimento de pesquisas e na obtenção de respostas mais concretas para a análise do impacto dos investimentos em TI nas organizações.

O objetivo deste artigo é analisar a efetividade de conversão dos investimentos em TI nos bancos brasileiros no período de 1995 a 1999, com base em fatores estratégicos. Para isto propõe-se novo modelo para avaliar a efetividade de conversão dos investimentos em TI nos bancos brasileiros, utilizando a técnica DEA.

Este trabalho está organizado da seguinte forma: a primeira parte apresenta uma revisão de estudos que aplicaram o DEA na análise da eficiência da TI na indústria bancária; a segunda parte apresenta o modelo de pesquisa adotado; o método de pesquisa é apresentado na terceira parte ; a quarta parte apresenta os resultados obtidos, discutidos na quinta parte; finalmente a sexta parte conclui o artigo. 


\section{DEA, tI e Análise da Eficiência na Indústria Bancária}

\section{DEA}

Apesar do grande número de pesquisas que aplica a técnica DEA nos últimos 20 anos, Schaffnit et al. (1997), Siems e Barr (1998) e Troutt et al. (1999) consideram esta técnica como forma atual e efetiva de visualizar e analisar a eficiência de processos complexos em DMU (do inglês, Decision Making Unit) que são as unidades de análise, como, por exemplo: bancos, hospitais, jogadores de futebol.

A justificativa para o crescente uso da técnica DEA é o conjunto de possibilidades de análise dos dados que a ferramenta disponibiliza. Algumas razões que motivaram o intenso uso da aplicação são: poucas técnicas disponíveis para avaliar a eficiência das organizações; confiança por parte dos pesquisadores nos resultados obtidos de sua utilização; facilidade de utilização; e existência de softwares que reduzem o esforço e facilitam a elaboração de cenários. Além disso, as simulações de cenários pela técnica DEA possibilitam identificar as organizações eficientes e as não eficientes. As organizações eficientes são consideradas aquelas que têm as melhores práticas e servem de modelos para pesquisas futuras e de análise de benchmarking (BERGENDAHL, 1998; SCHAFFNIT et al., 1997; SIEMS; BARR, 1998).

Wang et al. (1997) apresentam algumas vantagens da aplicação da técnica DEA como ferramenta empírica, apoiando-se em pesquisa que buscou identificar e analisar o impacto da TI sobre a eficiência organizacional, foco deste trabalho. As vantagens são: (1) os dados sobre os investimentos em TI não necessitam ser normalizados; em contraposição, a normalização é necessária em abordagens econométricas; (2) a técnica DEA é uma abordagem não-paramétrica, não exigindo forma funcional explícita que relacione input e output; uma abordagem paramétrica requer forma funcional explícita que relacione input e output, para a qual é necessário um conhecimento detalhado do processo de produção; (3) a técnica DEA indica explicitamente a eficiência do processo de produção relacionado com a TI; (4) a técnica DEA, diferentemente de modelos econométricos, captura mais facilmente constructos qualitativos que refletem aspectos comportamentais ou de percepção de envolvimento dos usuários da TI; tais fatores, segundo os autores, têm sido sugeridos na literatura como as medidas mais apropriadas da incorporação da TI nas organizações.

Conforme Schaffnit et al. (1997), a técnica DEA também oferece vantagens em relação aos métodos tradicionais de avaliação de eficiência, como análise de regressão e de índices. Thanassoulis (1993) e Athanassopoulos e Curram (1996) 
confirmam em seus estudos as vantagens do uso da técnica DEA em relação a outras técnicas de avaliação de eficiência, como redes neurais e análise de regressão.

\section{DEA, TI e Bancos}

A técnica DEA é citada por Bakos e Kemerer (1992) como uma das técnicas baseadas na teoria econômica, sendo utilizada por vários pesquisadores com o objetivo de (1) estudar a capacidade da TI; (2) estudar seus efeitos na eficiência organizacional; e (3) analisar a eficiência organizacional. Na indústria bancária, entretanto, poucos estudos são encontrados na literatura nas últimas décadas com aplicação da técnica DEA para analisar e medir a eficiência dos investimentos em TI. A revisão revela lacunas e possibilidades de desenvolvimento de novos modelos para análise da eficiência dos investimentos em TI nos bancos.

Tulkens (1993) conduziu sua pesquisa estudando 773 agências bancárias que operam na Bélgica. Dentre os fatores utilizados, apenas um fator de input e um de output estão relacionados com TI (número de máquinas ATM e número de operações intermediadas por máquinas ATM). Nenhuma análise que enfoque a eficiência desses fatores é citada no estudo.

Mahmood (1994) investigou o impacto dos investimentos em TI, aplicando a técnica DEA, usando uma amostra de 81 empresas citadas pela revista Computerworld como as que mais investem em TI, incluindo bancos. O autor aponta a existência de relação entre os investimentos em TI com a estratégia organizacional e eficiência econômica, salientando a necessidade de um estudo longitudinal para uma melhor consistência das análises.

O estudo desenvolvido por Wang et al. (1997) disponibiliza uma avaliação da eficiência dos investimentos em TI nos bancos americanos, utilizando a técnica DEA. Deste estudo destacam-se dois pontos, que devem ser levados em consideração no desenvolvimento de pesquisas que envolvem essa temática, como: o impacto da TI é indireto e, conseqüentemente, os impactos da TI devem ser observados e analisados a partir de processos de produção intermediários. Estes pontos levaram os autores a desenvolver um modelo de produção de dois estágios com o objetivo de mapear em que medida os processos intermediários afetam a eficiência dos bancos. Para melhor uso da técnica, os autores recomendam que o estudo utilize dados que possibilitem avaliar diferentes períodos, já que existe um intervalo de tempo entre os investimentos em TI realizados e a eficiência organizacional.

Alpar e Porembski (1998) utilizam a técnica DEA para analisar os impactos dos investimentos em TI em uma amostra de 30 bancos alemães, classificados como pequenos (patrimônio entre 0,2 a 6 bilhões de marcos alemães). $O$ modelo 
de produção calculou a eficiência do período entre 1989 e 1994. Os bancos foram divididos em três grupos, sendo que as organizações do grupo intermediário (1 a 3 bilhões de marcos alemães) foram identificadas como as mais eficientes.

A pesquisa desenvolvida por Banker e Ou (1998) não trata os investimentos em TI como um fator principal. Os investimentos em TI são incluídos no input custos de apoio, juntamente com outros custos operacionais. São feitas análises de eficiência de 1982 a 1988. O escopo da pesquisa foi identificar quais os impactos dos investimentos em TI na redução dos custos operacionais, pois os autores consideram que os produtos e serviços e as redes de agências são os responsáveis por esses custos. Os investimentos em TI aparecem somente na razão número de ATM por número de contas corrente. A principal conclusão dos autores é que as despesas operacionais foram diminuindo com o uso intensivo da TI.

O estudo publicado por Shafer e Byrd (2000) apresenta um modelo de transformação elaborado a partir de input e output, para analisar a eficiência organizacional dos investimentos em TI. Os dados foram selecionados do ranking dos Best Users of IT, da revista Computerworld, constituído por vários tipos de organizações, incluindo os bancos. O diferencial dessa investigação é a preocupação dos autores em discutir a validade dos resultados fornecidos pela técnica DEA.

Cabe ressaltar que apenas Mahmood (1994) e Shafer e Byrd (2000) utilizaram em suas pesquisas três input relacionados com investimentos em TI. O Quadro 1 apresenta os input e output dos estudos revisados. Os input e output apresentados no Quadro 1 representam um esforço que abrange o objetivo da pesquisa e a disponibilidade de dados. Em alguns estudos, o fator depósito aparece como input e output. Percebe-se essa dualidade em estudos que se compõem de dois ou mais estágios. Wang et al. (1997) e Banker e Ou (1998) utilizam o fator depósitos como output (abordagem de produção) no primeiro estágio, e, no segundo estágio, como input e possível gerador de receitas vinculadas a produtos e serviços (abordagem de intermediação). Pastor et al. (1997) revelam que muitas pesquisas tratam os depósitos como input e output, e que novos estudos têm procurado resolver o problema de identificação do papel desse fator de diferentes maneiras. Por exemplo, Berger et al. (1993) evitam o problema de identificação, analisando o problema de eficiência por meio da função lucro, o que fixa os depósitos como apenas mais um recurso.

Mukhopadhyay et al. (1997) salientam que o fato da TI atuar nos processos das organizações implica examinar o seu impacto, por meio de vários estágios de transformação. Conforme Barua e Mukhopadhyay (2000), a análise do impacto da TI sobre processos intermediários é de suma importância para as organizações. Isto colabora para que os modelos de análise de eficiência sejam desenvolvidos considerando mais de um estágio, a fim de avaliar os impactos dos investimentos em TI. 


\section{Quadro 1: Aplicações da Técnica DEA em Bancos com Fatores de Investimentos em TI}

\begin{tabular}{|c|c|c|}
\hline Autores & Input & Output \\
\hline Tulkens (1993) & $\begin{array}{ll}- & \text { número de terminais ATM; } \\
\text { - } & \text { gastos com pessoal; } \\
\text { - } & \text { marketing; } \\
\text { - } & \text { número de contas; } \\
\text { - } & \text { juros de aplicações de crédito. }\end{array}$ & $\begin{array}{l}\text { - } \quad \text { transações de clientes; } \\
\text { - } \quad \text { receitas. }\end{array}$ \\
\hline Mahmood (1994) & 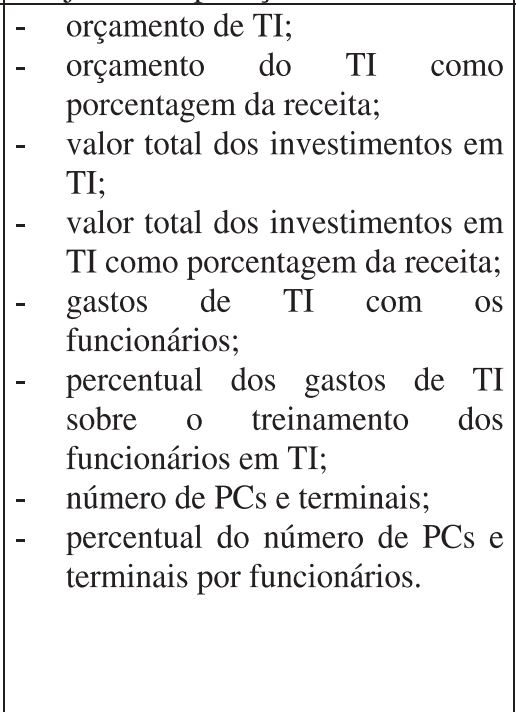 & $\begin{array}{ll}\text { - } & \text { retorno sobre vendas; } \\
\text { - } & \text { crescimento de } \\
& \text { receitas; } \\
\text { - } & \text { vendas sobre } \\
& \text { patrimônio; } \\
\text { - } & \text { dividendos pagos; } \\
\text { - } & \text { vendas por } \\
& \text { empregado; } \\
\text { - débito total sobre } & \\
\text { patrimônio; } \\
\text { - capital sobre receitas } \\
\text { de vendas; } \\
\text { - retorno } \\
\text { investimento; } \\
\text { - valor de mercado } \\
\text { contábil; } \\
\text { fluxo de caixa do } \\
\text { investimento. }\end{array}$ \\
\hline Wang et al. (1997) & $\begin{array}{l}\text { Estágio I } \\
-\quad \text { gastos com TI; } \\
-\quad \text { patrimônio líquido; } \\
-\quad \text { número de funcionários; } \\
\text { Estágio II } \\
-\quad \text { depósitos. }\end{array}$ & $\begin{array}{l}\text { Estágio I } \\
\text { - } \quad \text { depósitos; } \\
\text { Estágio II } \\
\text { - } \quad \text { resultado líquido; } \\
\text { - } \quad \text { empréstimos } \\
\quad \text { recuperados. }\end{array}$ \\
\hline $\begin{array}{l}\text { Alpar } \\
(1998)\end{array}$ & $\begin{array}{ll}- & \text { gastos com TI; } \\
- & \text { número de empregados; } \\
- & \text { patrimônio. }\end{array}$ & $\begin{array}{l}\text { - número de contas } \\
\text { (três diferentes tipos } \\
\text { de contas). }\end{array}$ \\
\hline Banker e Ou (1998) & $\begin{array}{ll}- & \text { custos com pessoal } \\
- & \text { custos com infra-estrutura; } \\
\text { - } & \text { custos operacionais (gastos com } \\
\text { TI) }\end{array}$ & $\begin{array}{l}\text { - } \quad \text { empréstimos; } \\
\text { - } \quad \text { depósitos. }\end{array}$ \\
\hline Shafer e Byrd (2000) & 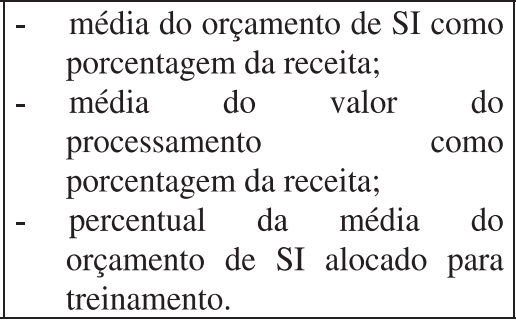 & $\begin{array}{ll}\text { - } & \text { vendas; } \\
\text { - } & \text { lucro. }\end{array}$ \\
\hline
\end{tabular}


Cabe destacar que, dos estudos revisados, apenas Siems e Barr (1998) formalizam o processo de seleção e validação dos fatores que fazem parte do modelo de análise de eficiência desenvolvido, aplicando uma survey junto a executivos de bancos. Pode-se afirmar que a maior parte das investigações segue as escolhas de input e output, respeitando apenas os objetivos de pesquisa e dos bancos, e a disponibilidade dos dados.

\section{Modelo de Pesquisa}

O modelo de pesquisa utilizado neste trabalho (ver Figura 1) é uma extensão dos modelos desenvolvidos por Trice e Treacy (1986); Weill (1989); McKeen e Smith (1996) e McKeen et al. (1999).

\section{Figura 1: Modelo de Pesquisa}

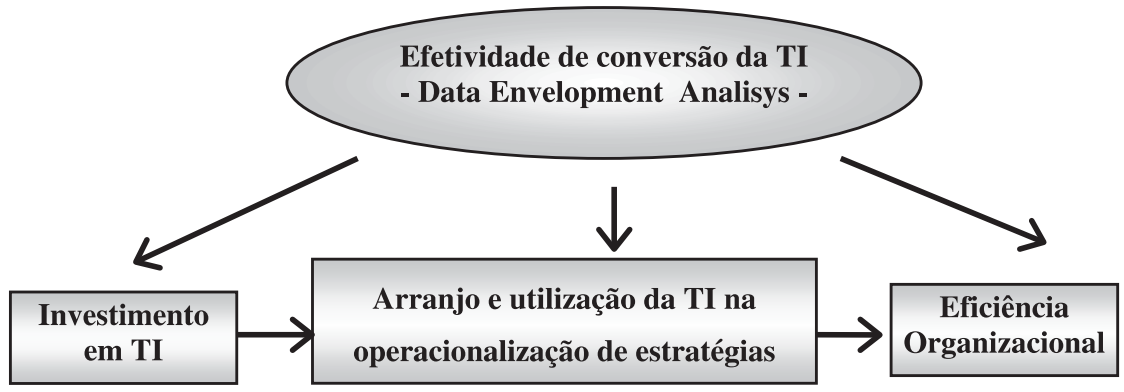

Os primeiros estudos sobre impacto dos investimentos em TI utilizaram o modelo básico de produção, modelando a transformação de input (investimento em TI) em output (eficiência organizacional). O modelo da Figura 1 dá destaque a medidas sobre variáveis estratégicas organizacionais relacionadas com o arranjo e utilização da TI. Venkatraman (1991) fundamenta esta inclusão por considerar que não tem sentido discutir se a TI é estratégica, mas se existe efetiva conversão da sua utilização na gestão e implementação de estratégias.

\section{Método}

O modelo CCR, desenvolvido por Charnes, Cooper e Rhodes (1978) é utilizado neste artigo. Na aplicação desse modelo pode-se optar pela orientação a input (obter o mínimo emprego de input dado o nível de output) ou orientação a output 
(obter o máximo nível de output mantendo o nível de input). Justifica-se a aplicação do modelo CCR orientado para input, pois a eficiência dos investimentos em TI nos bancos é observada a partir do input investimentos em TI e de como esse fator auxilia na transformação de output.

A medida de eficiência usualmente utilizada é uma razão entre uma soma ponderada de output e uma soma ponderada de input (SIEMS, 1992):

$\operatorname{EFICIÊNCIA~}_{k}=\frac{\sum_{j=1}^{n} W_{j k} \text { OUTPUT }_{j k}}{\sum_{i=1}^{m} V_{i k} \text { INPUT }_{i k}} \mathrm{k}=1, \ldots, \mathrm{N}$,

onde $V_{i k}$ é o peso unitário do input i e $W_{j k}$ é o peso unitário do output j para a unidade estudada $k$. Sob esta notação, há $N$ unidades estudadas, $m$ variáveis de input e $n$ variáveis de output. Para cada unidade estudada, determina-se o conjunto de pesos que lhe dá maior eficiência possível. Pode-se notar que a eficiência é usualmente restringida entre os limites 0 e 1 . A definição operacional de input e output depende do contexto e dos objetivos do problema de pesquisa, não tendo função de produção predefinida idêntica para todas as organizações na análise do relacionamento insumo-produto-eficiência. Avalia-se a eficiência de cada DMU, comparando-a a um grupo de referência, constituído por outras DMUs com o mesmo conjunto de input e output (SAHA; RAVISANKAR, 2000). Uma DMU é definida como eficiente (taxa de eficiência igual a 100\%), se nenhuma outra DMU no conjunto de dados puder produzir mais output com o mesmo input, ou o mesmo output utilizando menos input (SOTERIOU; ZENIOS, 1999).

Assim como é válido para a aplicação de qualquer abordagem analítica da arte da avaliação, o uso da técnica DEA requer conhecimento a respeito da formulação de modelos, escolha de fatores, hipóteses subjacentes, representação de dados, interpretação de resultados e conhecimento de suas limitações (CHARNES et al., 1994). Schaffnit et al. (1997) descrevem que dois pontos são cruciais e devem ser observados para a utilização da técnica: a elaboração de um modelo de produção e a escolha de input e output relevantes e relacionados para suprir o modelo. Algumas experiências apresentadas por pesquisadores revelam que a seleção adequada de fatores de input e de output é a questão mais importante na utilização da técnica DEA para medir a eficiência de qualquer tipo de firma, uma vez que determina o foco de avaliação da comparação (YEH, 1996). A seleção de fatores é uma tarefa que deve ser desenvolvida com constante feedback, ou seja, não há exatamente uma conduta linear a ser seguida. Para Golany e Roll (1989), a aplicação do método deve também considerar três fases 
no estudo de medida de eficiência: definição e seleção das DMU para análise, determinação de fatores de input e output que são relevantes e apropriados, e elaboração de um modelo de análise de eficiência.

Neste trabalho, as DMU analisadas representam uma amostra (intencional) composta por 41 bancos brasileiros (de um total de 125 filiados à FEBRABAN). Os dados extraídos dos balanços dos bancos referem-se ao período de 1995 a 1999. A escolha por esse período de análise, após o Plano de Estabilização Econômica (Plano Real), implantado em 1994, possibilitou que o estudo utilizasse dados com a mesma unidade monetária. Os bancos que compõem esta amostra representam 68\% do patrimônio líquido total dos bancos brasileiros associados à FEBRABAN. A seleção dos input e output foi realizada em várias etapas. Iniciouse com uma pesquisa exploratória, usando a técnica de entrevistas com 5 executivos da Comissão Nacional de Automação Bancária da FEBRABAN, para identificar o papel da TI nas estratégias dos bancos brasileiros. A seguir realizouse uma pesquisa tipo survey, visando estabelecer um conjunto de variáveis estratégicas organizacionais influenciadas pela TI, baseado em Mahmood (1993) e Palvia (1998). Nesta etapa 125 bancos receberam os questionários. Retornaram 141 questionários de 68 bancos, com representatividade de 79\% do patrimônio líquido daqueles 125 bancos previamente selecionados. Os respondentes são executivos de TI e da área administrativa. A lista de variáveis foi confirmada por procedimentos estatísticos de análise confirmatória (equações estruturais). As variáveis resultantes foram então adaptadas como input e output observáveis em contas de balanço por contadores de bancos filiados à FEBRABAN. Os dados de balanço dos bancos selecionados foram fornecidos pelo Banco Central do Brasil.

O modelo desenvolvido é composto por dois estágios, com quatro fatores de input, dois fatores que de input e output e três fatores de output. Os parâmetros do modelo DEA foram estimados, utilizando o software DEA Warwick ${ }^{\circledR}$, especificamente o modelo CCR.

A presente investigação visa responder à seguinte questão de pesquisa: quais são os impactos dos investimentos em Tecnologia de Informação nas variáveis estratégicas e na eficiência dos bancos brasileiros?

\section{Resultados}

A seguir apresentam-se os resultados obtidos com a investigação. Inicialmente, na primeira e segunda seções, os resultados da modelagem são apresentados: 
seleção de fatores e desenvolvimento do modelo de eficiência. Em seqüência, na terceira seção, apresentam-se os resultados da aplicação do modelo à amostra selecionada.

\section{Seleção de Fatores}

\section{Entrevistas com Executivos}

De uma maneira geral, as entrevistas com os executivos confirmaram, segundo os tópicos revisados na literatura de SI, a importância da TI e o seu papel na implementação das estratégias nas organizações e, especificamente, nos bancos brasileiros. No que diz respeito à aplicabilidade dos constructos ao contexto da pesquisa, os executivos consideraram-nos todos relacionados com o tema da pesquisa e com a utilização da TI. Os entrevistados confirmaram sua percepção de que os investimentos em TI produzem impactos nas variáveis estratégicas organizacionais.

Um aspecto, em especial, apontado pelos executivos foi a abordagem multidimensional oferecida no conjunto de constructos apresentados, permitindo medir os impactos dos investimentos em TI nas várias dimensões da organização. Segundo os entrevistados, as análises de impacto de TI desenvolvidas até o presente momento estavam apoiadas por medidas unidimensionais, não permitindo uma análise mais ampla dos efeitos da TI.

\section{Pesquisa Survey}

A pesquisa survey resultou em um conjunto de variáveis estratégicas afetadas pela TI (Quadro 2). Os procedimentos metodológicos desenvolvidos confirmaram 12 variáveis, baseando sua mensuração em 35 itens. O coeficiente de fidedignidade (alfa de Cronbach) do instrumento atingiu 0,90. Os coeficientes das variáveis que o compõem situam-se entre 0,71 e 0,95. A validade discriminante e a unidimensionalidade dos constructos foi assegurada. O modelo de mensuração foi confirmado com um índice de qualidade de ajustamento (GFI - do inglês goodness of fit index) igual a 0,90. 
Quadro 2: Variáveis Estratégicas Identificadas

\begin{tabular}{|l|}
\hline Variáveis \\
\hline 1.Tomadores de Recursos Financeiros (TRF) \\
\hline 2.Competitividade (COM) \\
\hline 3.Fornecedores de Recursos Financeiros (FRF) \\
\hline 4.Coleta e Troca de Informações (CTI) \\
\hline 5.Produtos e Serviços (Pés) \\
\hline 6.Estrutura de Custos e Capacidade (ECC) \\
\hline 7.Eficiência Organizacional Interna (EOI) \\
\hline 8.Eficiência Inter-organizacional (EIN) \\
\hline 9.Preços (PRE) \\
\hline 10.Internacionalização (INT) \\
\hline 11.Requisitos de Governos e Países (RGP) \\
\hline 12. Coordenação Inter-organizacional (CIN) \\
\hline
\end{tabular}

A variável competitividade expressa genericamente o que a literatura de SI apresenta como principal fator para utilização da TI (CALLAHAN; NEMEC JR., 1999; PORTER; MILLAR, 1985; ROCKART et al., 1996). As demais variáveis identificadas, todas, em certa medida, ligadas à noção geral de competitividade, segmentam aspectos mais específicos ligados à operação dos bancos. Os resultados refletem o atual contexto competitivo em que os bancos se inserem, com perspectivas de fusões e aquisições tanto internacionais como nacionais (SIJBRANDS; EPPINK, 1994; ALBRIGHT; PARKER, 1998). Por outro lado, a TI, particularmente com máquinas ATM e Internet, tem possibilitado o desenvolvimento e distribuição de novos produtos e serviços diferenciados, conquistando os clientes (DEVLIN, 1995; LUCAS JR., 1999; JORDAN; KATZ, 1999).

\section{Adaptação em Input e Output Observáveis em Contas de Balanço}

O processo de adaptação dos constructos em fatores de input e output, identificados em contas de balanço, para suprir o modelo de análise de eficiência deu-se por meio de entrevistas em grupo com cinco contadores selecionados pela FEBRABAN. Na realização desta etapa, foram utilizados alguns documentos para subsidiar os executivos no processo de adaptação dos constructos em input e output: lista dos constructos e o seu significado, um modelo de análise de eficiência de referência desenvolvido por Wang et al. (1997), com o objetivo de exemplificar como cada input e output pode ser posicionado no modelo em desenvolvimento e o Plano Contábil das Instituições do Sistema Financeiro Nacional (SFN) - COSIF - Circular BCB n 1273 (www.cosif.com.br). 
A escolha das contas de balanço como fonte de dados deu-se devido à dificuldade na obtenção de informações específicas, como os valores dos investimentos em TI, essencial para o desenvolvimento desta pesquisa. Esta é considerada uma informação estratégica pelos executivos dos bancos, e seus valores usualmente não são disponibilizados. Também foi necessário adaptar as variáveis estratégicas organizacionais (ver Quadro 2), em input e output observáveis em contas de balanço (ver Quadro 3) que representassem fatores estratégicos para os bancos. Conforme Bergendahl (1998), os bancos são organizações que dificultam o acesso a informações sobre investimentos em TI. O autor considera o balanço a única fonte para fornecer os dados para suprir modelos de análise de eficiência elaborados para a indústria bancária.

O quadro 3 apresenta o conjunto de contas correspondente à variável Investimento em TI. O resultado aritmético das operações mencionadas é o valor dos investimentos em TI.

\section{Quadro 3: Conjunto de Contas do Valor dos Investimento em TI}

\begin{tabular}{|ll|}
\hline ( + ) 2.2.9.10.00-6 & Sistemas de Comunicação \\
( + ) 2.2.9.30.00-0 & Sistema de Processamento de Dados \\
( + ) 2.4.1.60.00-5 & Gastos com Aquisição e Desenvolvimento de Logiciais \\
( - ) 2.2.9.99.10-6 & Depreciação Acumulada de Sistemas de Comunicação - Equipamentos \\
( - ) 2.2.9.99.30-2 & Depreciação Acumulada de Sistemas de Processamento de Dados \\
( - ) 2.2.9.99.60-5 & Amortização Acumulada do Diferido Relativa aos Gastos com \\
& Aquisição e Desenvolvimento de Logiciais \\
\hline
\end{tabular}

A conta Sistemas de Comunicação registra os valores dos equipamentos de comunicação e direitos de uso, como centrais telefônicas, telex e outros de propriedade da instituição. O valor dos equipamentos que compõem o sistema de processamento eletrônico de dados de propriedade da instituição é registrado na conta Sistema de Processamento de Dados. Podem ser também registrados nesta conta os valores correspondentes a instalações, entendidas apenas como as transformações no imóvel que se fizerem necessárias ao perfeito funcionamento da aparelhagem eletrônica, bem como todas as despesas necessárias à sua aquisição, tais como transporte, seguro e tributo.

Na conta Gastos com Aquisição e Desenvolvimento de Logiciais são registrados os custos e despesas de aquisição e desenvolvimento de logiciais (software), utilizados em processamento de dados.

As parcelas negativas na equação referem-se a contas que compõem o título Depreciação Acumulada de Outras Imobilizações de Uso. Nos seus subtítulos 2.2.9.99.10-6, 2.2.9.30.00-0 e 2.4.1.60.00-5 registram-se os valores das depreciações acumuladas de bens do imobilizado, em uso na exploração da 
atividade social, relativos a Sistemas de Comunicação e de Processamento de Dados (hardware e software).

Após a definição do input investimento em TI, usando o mesmo método, os executivos iniciaram o processo de adaptação em input e output do conjunto de constructos enumerados no Quadro 2. Tal processo desenvolveu-se simultaneamente com o desenvolvimento do modelo de análise, explicitado na próxima seção. Para detalhes da identificação dos fatores nas contas dos balanços, ver Maçada (2001).

\section{Modelo de Eficiência Desenvolvido}

O trabalho se apóia no modelo de análise de eficiência apresentado na Figura 2. Participaram do processo de definição do modelo os executivos da FEBRABAN. Os executivos definiram o primeiro estágio do modelo como de produção (transformação de recursos), utilizando os input investimentos em TI, despesas com pessoal, outras despesas e despesas de internacionalização na produção dos output ativos de transações financeiras e outros ativos. Para os executivos, o estágio 1 representa a capacidade de o banco ser competitivo.

\section{Figura 2: Modelo de Análise de Eficiência}

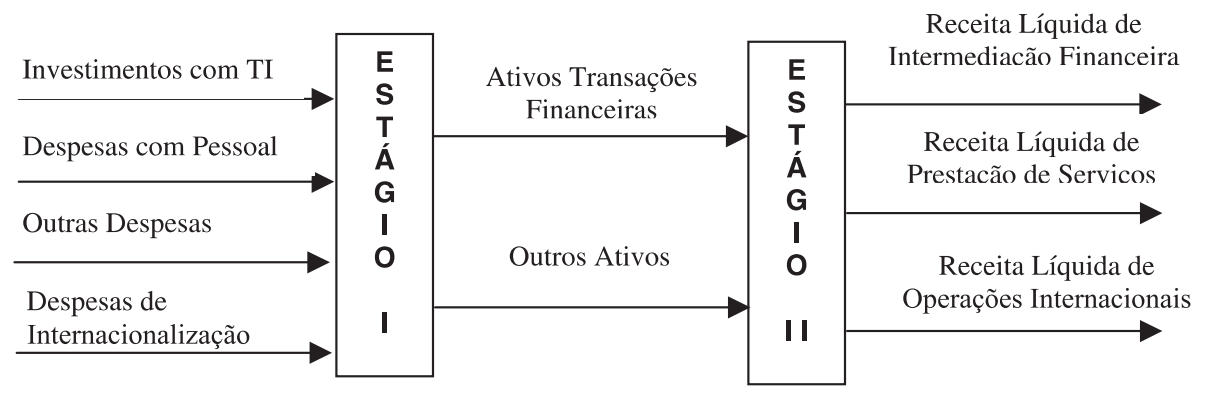

Os executivos revelaram também a importância da inclusão de um segundo estágio de produção, tendo como input os ativos de transações financeiras e outros ativos como recurso para gerar receitas de intermediação financeira, de prestação de serviços e de operações internacionais. Os índices obtidos na análise global representam a transformação dos input do primeiro estágio (como recursos primários) em output do segundo estágio (como resultados finais).

O modelo desenvolvido pressupõe que os investimentos em TI, combinados com outros fatores de input (despesas com pessoal, outras despesas administrativas e despesas de internacionalização) transformam-se em output (receitas líquidas de intermediação financeira, de prestação de serviços e de operações 
internacionais) de maneira indireta, usando o conceito desenvolvido por Weill (1989) de efetividade de conversão. O nível de efetividade de conversão representa o mix do gerenciamento interno e os fatores ambientais externos. O modelo acima, desenvolvido especialmente para a indústria bancária, salienta que os investimentos em TI gerarão incremento nas receitas, mas esta relação é intermediada pela acumulação de ativos, financeiros e não financeiros. A capacidade dos bancos gerarem ativos é considerada um fator importante de competitividade (Banco Hoje, 2/7/2000). Cabe salientar que o fator investimento em TI é combinado com outros, pois a TI não pode ser aplicada e utilizada sem a participação das pessoas (e as despesas decorrentes) que a utilizam no processo de tomada de decisões, dando suporte a uma estratégia de orientação (neste caso a estratégia de internacionalização).

\section{Análise dos Resultados}

Os resultados são apresentados nos Quadros 4 e 5 . A amostra da pesquisa foi segmentada por porte (P: pequeno; M: médio; G: grande) e capital (PrN: privado nacional; PrNPE: privado nacional com participação estrangeira; E: estrangeiro; PrNCE: privado nacional com controle estrangeiro; PuF: público federal; PuE: público estadual), segundo classificação do Banco Central do Brasil e utilizada pela FEBRABAN. 


\begin{tabular}{|c|c|c|}
\hline बे & 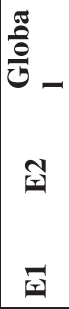 & 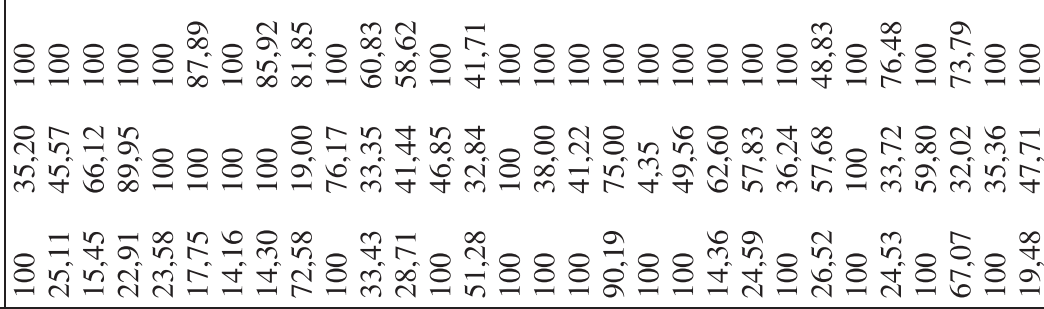 \\
\hline & $\begin{array}{l}\frac{\sigma}{0} \\
\frac{0}{0} \\
\text { ป }\end{array}$ & 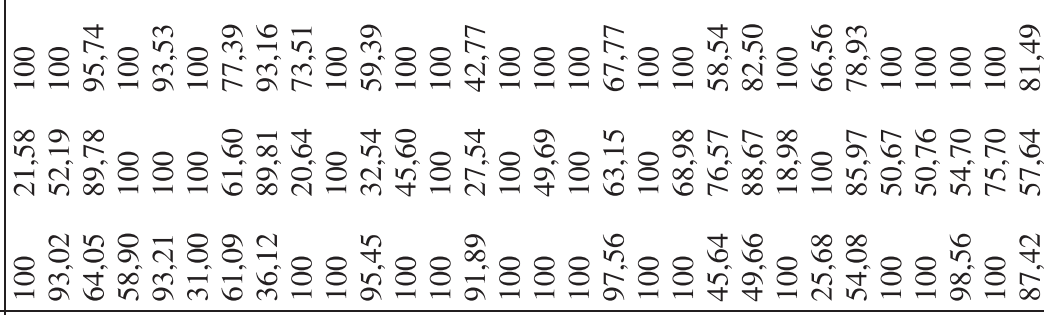 \\
\hline$\hat{\mathrm{z}}$ & _ & 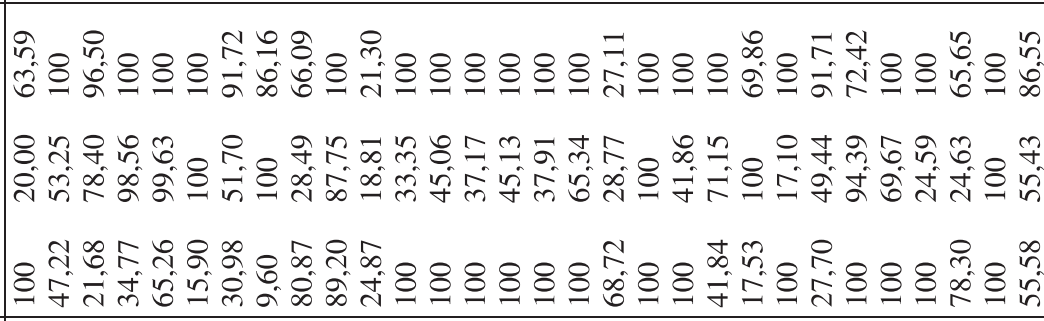 \\
\hline ڤั & $\begin{array}{l}\frac{\pi}{0} \\
\frac{0}{0} \\
\mathbb{0}\end{array}$ &  \\
\hline 2 & 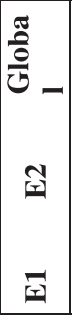 & 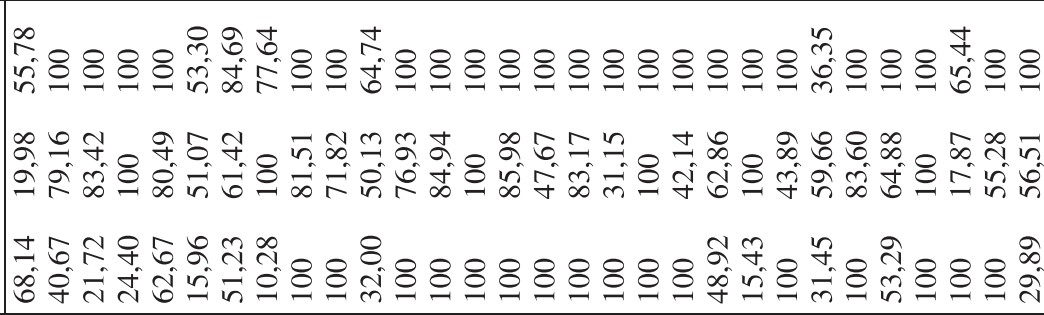 \\
\hline & ט.: & 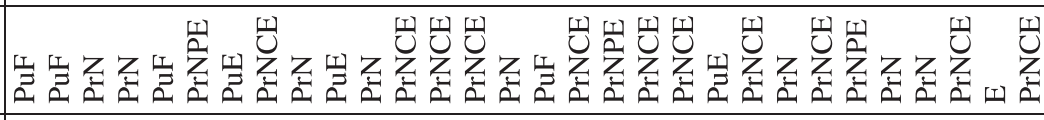 \\
\hline & : & ט \\
\hline & $\stackrel{\Xi}{\exists}$ & \\
\hline
\end{tabular}




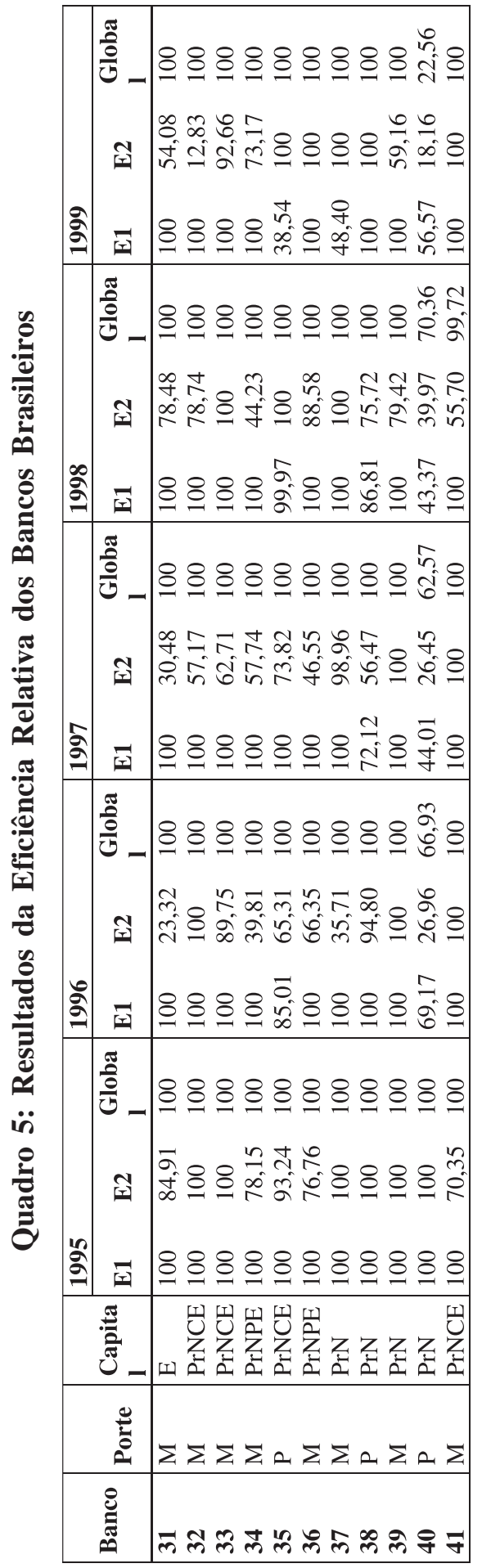




\section{Dıscussão}

De uma maneira geral, os bancos revelam-se eficientes globalmente (combinando os estágios 1 e 2). O índice médio de eficiência global é de 93,61\% em 1995, 91,14\% em 1996, 90,27\% em 1997, 91,25\% em 1998, e 91,18\% em 1999. Em média 72,2\% dos bancos apresentam índice de eficiência igual a 100\% ao longo do período analisado. Este índice percentual varia ao longo do período, de 82,9\% em 1995, 70,7\% em 1996, 68,3\% em 1997, 63,4\% em 1998, e 75,6\% em 1999. Observa-se que em 1996 o volume total de investimentos em TI diminuiu, passando de 1.791 milhões de reais em 1995 para 1.615 milhões de reais em 1996. Isto deve ter provocado efeitos na eficiência global dos bancos. A partir de 1997, os investimentos em TI apresentaram uma tendência de crescimento, totalizando 1.718 milhões de reais em 1997, 1.994 milhões de reais em 1998, e 2.792 milhões de reais em 1999. Os efeitos deste crescimento se fizeram perceber apenas em 1999, reforçando as observações de Brynjolfson e Hitt (1998) quanto à defasagem entre investimentos em TI e seus decorrentes resultados.

Os nove bancos que mais investiram em TI no período analisado (bancos 1, 2, $3,4,5,6,8,13$, e 22, todos grandes, com exceção do banco 22, que é médio) realizaram investimentos médios superiores a 40 milhões de reais ao ano. Uma análise deste conjunto de bancos quanto ao capital revela sua diversidade, sendo formado por três bancos públicos federais, dois bancos privados nacionais, três bancos privados nacionais com controle estrangeiro e um banco privado nacional com participação estrangeira. Apesar de os bancos privados nacionais serem menos numerosos, o volume de seus investimentos em TI é bastante representativo (32,5\% em relação ao total da amostra). Também se destacam os bancos públicos federais, com participação percentual no volume de investimentos em TI de 26,2\% em relação ao total da amostra. Os bancos com participação ou com controle estrangeiro representam 20,3\% do montante dos investimentos.

Os bancos deste segmento apresentam índices de eficiência global de 100\% em 1999, com exceção dos bancos 6 e 8. Estes apresentam índices de eficiência global respectivamente de $87,89 \%$ e $85,92 \%$ em 1999, bastante próximos da eficiência máxima. Percebe-se que o banco 6 apresenta uma eficiência global de apenas 53,30\% em 1995, aumentando sua eficiência ao longo do período analisado, atingindo eficiência máxima em 1997 e 1998. Já o banco 8, por sua vez, apresenta eficiência global de 77,64\% em 1995, aumentando estavelmente seu índice de eficiência ao longo do período analisado, atingindo 93,16\% em 1998. O índice médio de eficiência global deste segmento da amostra é de 87,41\% em 1995, 93,14\% em 1996, 90,67\% em 1997, 96,10\% em 1998, e 97,09\% em 1999. 
Comparando-se com os índices médios de eficiência global da amostra completa de 41 bancos, percebe-se claramente os efeitos dos altos investimentos em TI na eficiência global dos bancos (Figura 3). Embora tenham realizado vultosos investimentos em TI em 1995, alguns bancos deste segmento aparentemente não conseguiram combinar estes investimentos com as demais variáveis estudadas de forma eficiente ainda naquele ano, mas os resultados em períodos subseqüentes são evidentes. O ciclo de vida das tecnologias de informação é, em geral, pequeno (MEIRELLES, 1996), gerando a necessidade de renovação da plataforma tecnológica em curtos espaços de tempo.

Figura 3: Eficiência Global Média ao Longo do Tempo

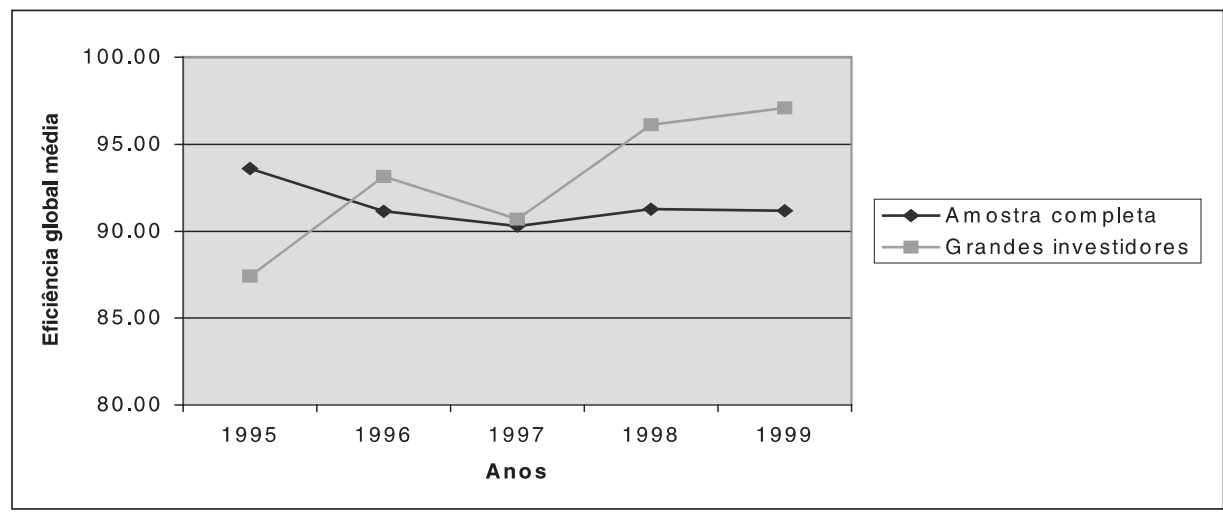

O segmento de bancos públicos (quatro públicos federais: 1, 2, 5 e 16; três públicos estaduais: 7, 10 e 21) revelam-se de uma maneira geral eficientes globalmente ao longo do período analisado. Esses bancos são de grande porte, estando entre os que mais investiram em TI entre 1995 e 1999 (913, 1.446, 233, 57, 151, 171 e 89 milhões de reais respectivamente). Todos apresentam-se eficientes globalmente em 1999, sendo que o banco 1 não foi eficiente em 1995 e em 1997; o banco 5 não foi completamente eficiente em 1998; o banco 7 apresenta oscilações em sua eficiência de 1995 a 1998; e o banco 21 não foi eficiente em 1998.

Sobre o banco 1 é importante ressaltar que seus investimentos em TI decaíram bastante em 1996 (28 milhões de reais em 1996 contra 129 milhões de reais em 1995), o que deve ter contribuído para a redução de sua eficiência em 1997. Já os investimentos em TI do banco oscilaram fortemente ao longo do período $(23,19$, 23, 46 e 40 milhões de reais respectivamente em 1995, 1996, 1997, 1998 e 1999), o que também deve ter contribuído para a oscilação verificada em sua eficiência.

Os quadros 2 e 3 também deixam transparecer que eficiência não é sinônimo de tamanho. De fato, 13 dos 15 bancos de médio porte (86,7\%) são eficientes 
globalmente em 1999, contra 16 dos 23 bancos grandes (69,6\%). Apesar de menores em relação aos demais, os investimentos em TI destes bancos, combinados com outras variáveis de input, parecem mais adequados aos seus objetivos, revelando melhor capacidade de gestão de investimentos e obtenção de resultados.

A análise de sensibilidade da solução fornecida pela técnica DEA oferece uma recomendação para cada DMU não eficiente com respeito às variáveis analisadas. Devido a limitações de espaço, não se apresentam aqui as recomendações fornecidas para todos os bancos da amostra, apenas alguns casos interessantes. Considere-se, por exemplo, o caso do banco menos eficiente em 1999 (banco 40). Este banco apresenta-se eficiente globalmente em 1995. Em 1996 sua eficiência caiu para 66,93\%, e a análise de sensibilidade aponta ser necessário reduzir seus investimentos em TI em 42,6\%, embora os investimentos não fossem tão elevados, comparativamente aos demais bancos (trata-se de banco de pequeno porte e de capital privado nacional). Ao invés disto, seus investimentos em TI aumentaram em 15\% de 1996 para 1997. Em 1997, sua eficiência global reduzse a 62,57\%, e a análise de sensibilidade realizada com os dados de 1997 reforça a necessidade de redução de 37,4\% em seus investimentos em TI. Em 1998 o banco manteve o mesmo volume de investimentos em TI em relação a 1997, e sua eficiência global melhorou um pouco, ficando em 70,36\%. A análise de sensibilidade realizada com os dados de 1998 novamente aponta para a necessidade de redução de 29,6\% em seus investimentos em TI. Em 1999, finalmente o banco reduziu seus investimentos em TI em cerca de 15\%, tarde demais, pois seu índice de eficiência despencou para 22,56\%. A análise de sensibilidade reforça a necessidade de redução de investimentos em TI em cerca de 77\%.

A análise longitudinal do banco 8 também é interessante. O banco apresenta índice de eficiência global de 77,64\% em 1995; a análise de sensibilidade aponta a necessidade de redução de 22,4\% em seus investimentos em TI (o banco é o sexto maior investidor em TI ao longo do período analisado). O banco reduziu levemente seus investimentos em TI em 1996, e sua eficiência eleva-se a 79,80\%. A análise de sensibilidade realizada com os dados de 1996 reforça a necessidade de redução dos investimentos em TI em 78,1\%. Em 1997 o banco reduz 5,7 milhões de reais em investimentos em TI (redução de 6,4\%), e sua eficiência global aumenta para 86,16\%. A análise de sensibilidade realizada com os dados deste ano ainda reforça a necessidade de redução de investimentos em TI (34,4\%). Em 1998 o banco corta mais 14,6 milhões de reais em investimentos em TI (redução de 17,4\%) e vemos sua eficiência global passar a 93,16\%. A análise de sensibilidade realizada com os dados de 1998 ainda aponta a necessidade de redução de investimentos em TI, embora com menor taxa percentual (6,8\%). Aparentemente em 1999 o banco altera sua estratégia, aumentando investimentos 
em TI em cerca de 23,3\%, e seu índice de eficiência baixa para 85,92\%. A análise de sensibilidade reforça a necessidade de redução de investimentos em TI em cerca de $14,1 \%$.

A análise da eficiência relativa nos estágios 1 e 2 também se revela interessante. De maneira geral, os bancos são menos eficientes no segundo estágio do que no primeiro, revelando maior capacidade de acumulação de ativos do que de transformação destes ativos em valores que possam trazer retornos para a organização. Comparando-se os índices médios de eficiência no primeiro estágio, percebe-se que os grandes investidores em TI são menos eficientes na geração de ativos relativamente ao conjunto total de bancos investigados, mas sua eficiência relativa aumentou no período analisado. Em contraste, a Figura 5 revela que os índices de eficiência no segundo estágio dos grandes investidores em TI são superiores aos do conjunto total de bancos analisados. Sua eficiência relativa tem permanecido estável ao longo do período analisado, contrapondo-se à tendência de queda observada para o conjunto de bancos. Depreende-se que os bancos desse segmento vêm ganhando, relativamente, eficiência na transformação de ativos em valor.

Cinco bancos destacam-se do conjunto analisado por obter eficiência máxima em todos os estágios no ano de 1999 (bancos 15, 25, 36, 38 e 41). Dois são de grande porte, dois são médios e um é pequeno. Todos são privados, dois nacionais, dois com participação estrangeira e um com controle estrangeiro. Todos têm obtido eficiência global quase máxima em todo o período analisado, tendo investido relativamente pouco em TI (média anual de 6,7 milhões de reais, com incremento nos últimos anos).

\section{Considerações Finais}

O modelo DEA é excelente ferramenta para avaliar o desempenho relativo de organizações. O modelo é bastante flexível, exigindo pouca preparação e formalização de dados. Sua utilização para comparar um determinado banco com seus concorrentes possibilita ao decisor aferir a operacionalização de suas estratégias, na forma de uma combinação de input para a obtenção de output. Sob uma ótica mais geral, conforme salienta Siems (1992), o modelo é de grande valia não somente para os administradores, mas também para os analistas, investidores e reguladores, podendo ser utilizado de várias maneiras no processo de avaliação.

Os escores obtidos pelo modelo DEA podem ser utilizados para segmentar a 
amostra dos bancos quanto à sua eficiência. A exploração de diferenças entre os segmentos, quanto a variáveis de interesse de analistas, investidores, administradores e reguladores, permite acumulação de conhecimento a respeito da indústria bancária. Isto se traduzirá, por exemplo, na formulação de melhores estratégias por parte de administradores, em investimentos mais sólidos e seguros por parte de investidores e no estabelecimento de controles mais acurados do sistema financeiro do país.

A análise dos resultados da indústria bancária brasileira permitiu observar que os bancos que investiram mais em TI ganharam eficiência ao longo do tempo. Os resultados revelam a necessidade de revisão das estratégias de aplicação dos investimentos em TI para vários bancos, com uma alteração nos cenários em estudo. A construção de cenários e de simulações com o auxilio do computador permitirá ao decisor identificar a combinação de input que proporciona níveis de output com a eficiência desejada.

\section{Artigo recebido em 20.12.2002. Aprovado em 22.06.2004.}

\section{Agradecimentos}

Os autores agradecem o apoio recebido para a elaboração da pesquisa que originou este trabalho das agências governamentais CNPq, CAPES e FAPERGS.

\section{REFERÊnCIAS BiblográficAs}

ALPAR, P.; POREMBSKI, $\mathrm{M}$.

Impact of IT on cost efficiency of German banks. Workshop on Information Systems and Economics, WISE’98, 1998. Disponível em: $<$ http://is-2.stern.nyu.edu/wise98/ program.html>. Acesso em: 20 de sept. 1998.

ANDERSON, T.

DEA: data envelopment analysis. Jan. 1996, p.1-5. Disponível em:
http://www.DEA.com>. Acesso em: 15 de mar. 1997

ATHANASSOPOULOS, A. D.; CURRAM S.P.

A comparison of data envelopment analysis and artificial neural networks as tools for assessing the efficiency of decision making units. Journal of the Operational Research Society, England, n.47, p.1000-1016, aug.1996. 
BAKOS, J. Y.;

KEMERER, C. F.

Recent applications of economic theory in information technology research. Decision Support Systems, Amsterdam, v. 8, n. 5, p. 365-386, sept. 1992.

BANDEIRA, D. L.

Análise da eficiência relativa de departamentos acadêmicos -0 caso da UFRGS. 2000. 133 f., Dissertação (Mestrado em Administração) Escola de Administração, Universidade Federal do Rio Grande do Sul, Porto Alegre.

BANKER, R. D.;

OU, C.S.

Product diversity and value of information technology: evidence from the u.s. banking industry. Workshop on Information Systems and Economics, WISE'98, 1998. Disponível em: <http:// is - 2. stern.nyu.edu/wis e 98 / program.html>. Acesso em: 20 de sept. 1988.

BANKER, R. D.;

CHARNES, A.;

COOPER, W. W.

Some models for estimating technical and scale inefficiencies in data envelopment analysis. Management Science, Baltimore, v. 30, n. 9, p. 10781092, 1092, sept. 1984.

BARUA, A.;

MUKHOPADHYAY, T.

Information technology and business performance: past, present and future. In: ZMUD, R. W.; PRICE, F. M. Framing the Domains of IT Management: projecting the future...through the past. Cincinnati,
Ohio: Pinnaflex Education Resources, USA, 2000. p. 65-84.

\section{BELTON, V.;}

VICKERS, S.P.

Demystifying DEA - a visual interactive approach based on multiple criteria analysis. Journal of the Operational Research Society. England, v. 44, n. 9, p. 883-896. sept. 1993.

\section{BERGENDAHL, G}

DEA and benchmarks - an application to nordic banks. Annals of Operations Research. Dordrecht, n. 82, p. 233-250, jan. 1998.

BERGER,A. N.;

HANCOCK, D.;

HUMPHREY, D. B.

Bank efficiency derived from the profit function. Journal of Banking and Finance, Amsterdam, v. 17, n. 2/3, p.317-347, apr. 1993.

BERGER,A. N.;

HUMPHREY, D. D.

Efficiency of financial institutions: international survey and directions for future research. European Journal of Operational Research, Amsterdam, v. 98, n. 2, p. 175-212, 16 apr.1997.

\section{BRYNJOLFSSON, E.;}

HITT, L. M.

Beyond the Productivity Paradox. Communications of the ACM, New York, v. 41, n. 8, p.49-55, aug., 1998.

BURLEY,H.T.;

DUCKETT,A.

Relative technical efficiency of New South Wales Public Hospital peer groups. Journal of Cost Analysis, Alexandria, v. 16, p.1-12, oct. 2000. 
CHARNES, A. et al.

Data envelopment analysis: theory, methodology and application. Massachusetts: Kluwer, 1994.

CHARNES, A.;

COOPER, W. W.;

RHODES.

Measuring efficiency of decision making units. European Journal of Operational Research, Amsterdam, v. 2, n. 6, p. 429-444, nov. 1978.

\section{CHIRIKOS, T. N.;}

SEAR, A. M.

Measuring hospital efficiency: a comparison of two approaches.

Health Services Research, Ann Arbor, v. 36, n. 6, p.1389-1408, Feb. 2000.

GOLANY,B.;

ROLL, Y.

An application procedure for DEA. Omega, Amsterdam, v. 17, n.3, p. 237250. 1989

\section{GROVER, V. et al.}

The influence of Information Technology diffusion and business process change on perceived productivity: the IS executive's perspective. Information and Management, Amsterdam, v. 34, n. 3, 1998, p.141-159.

\section{GUROVITZ, H.}

Falta de medida: poucas empresas sabem se gastam bem com Tecnologia da Informação, Exame, São Paulo, v. 35, n. 8, p. 40, 18 abr. 2001.
LAPA, A. L. M.;

LAPA, J. S.;

LANZER, E.A.

Análise envoltória de dados aplicada à avaliação de instituições de ensino superior: determinação de pesos relativos e valoração dos insumos e produtos. In: SIMPÓSIO BRASILEIRO DE PESQUISA ORGANIZACIONAL, 27., 1995, Vitória, Anais... Vitória: Sobrapo, 1995. p. 1-12.

MAÇADA, A. C. G.

Impacto dos investimentos em tecnologia de informação nas variáveis estratégicas e na eficiência dos bancos brasileiros. 2001. $198 \mathrm{f}$.. Tese (Doutorado em Administração) - Universidade Federal do Rio Grande do Sul, UFRGS, Rio Grande do Sul.

\section{MAHMOOD, M. A.}

Evaluating organizational efficiency resulting from information technology investment: an application of data envelopment analysis. Journal of Information Systems, Sarasota, v. 4, n. 2, p. 93-115, 1994.

\section{MAHMOOD, M. A.;}

MANN, G. J.

Special issue: impacts of information technology investment on organizational performance. Journal of Management Information Systems, Armonk, v. 17, n. 1, p. 3-10, Spring 2000.

\section{MUKHOPADHYAY, T;}

LERCH, F. J.;

MANGAL, V.

Assessing the impact of information technology on labor productivity: a field study. Decision Support System, Amsterdam, v. 19, n. 2, p.109-122, feb. 1997. 
PALMER, J. W.;

MARKUS, M. L. T

The performance impacts of quick response and strategic alignment in specialty retailing. Informations Systems Research, Linthicum, v. 11, n. 3, sep. 2000, p. 241-259.

PASTOR, J. M.;

PÉREZ, F;

QUESADA, J.

Efficiency analysis in banking firms: an international comparison. European Journal of Operational Research, Amsterdam, v. 98, n. 2, p. 395-407, 16 apr.1997.

PRADO, V. J.

Avaliando a eficiência das lojas da ECT do Rio Grande do Sul. 2000. 117

f. Dissertação (Mestrado em Administração) - Escola de Administração, Universidade Federal do Rio Grande do Sul, Porto Alegre.

SAHA, A.;

RAVISANKAR, T. S.

Rating of Indian commercial banks: a DEA approach. European Journal of Operational Research, Amsterdam, v. 124, n. 1, p. 187-203, july 2000.

SCHAFFNIT, C.;

ROSEN, D.;

PARADI, J. C.

Best practice analysis of bank branches: an application of DEA in a large Canadian bank. European Journal of Operational Research, Amsterdam, v. 98, n. 2, p. 269-289, 16 apr.1997.

\section{SHAFER, S. M.;}

BYRDT.A.

A framework for measuring the efficiency of organizational investments in information technology using data envelopment analysis. Omega, Dearbom, v. 28, n. 2, p. 125-141, apr. 2000.

SIEMS, T. F.;

BARR, R. S.

Benchmarking the productive efficiency of U.S. banks. financial industry studies, Federal Reserve Bank of Dallas, Dallas, p.11-24, dec., 1998.

\section{SOTERIOU,A.;}

\section{ZENIOS, S.}

Using data envelopment analysis for costing bank products. European Journal of Operational Research, Amsterdam, v. 114, n. 2, p.234-248, 16 apr. 1999.

THANASSAOULIS, E.;

DUSTAN, P.

Guiding schools to improved performance using data envelopment analysis: an illustration with data from a local education authority. Journal of the Operational Research Society. Engalnd, v. 45, n. 11, p. 1247-1262, nov. 1994.

THANASSOULIS, E.

A comparison of regression analysis and Data Envelopment Analysis as alternative methods for performance assessments. Journal of the Operational Research Society, England, v.44, n. 11, p.1129-1144, nov. 1993.

Introduction to the Theory and Application of Data Envelopment Analysis. Kluwer Academic Publisher, 2001. 
TROUTT, M.D. et al.

A new efficiency methodology for IT investment analysis studies. In: MAHMOOD, M. A. e SZEWCZAK, E. J. Measuring information technology investment payoff: contemporary approaches. Idea Group Publishing, Hershey, 1999. p. 202-222.

\section{TULKENS, $\mathrm{H}$.}

On FDH efficiency analysis: some methodological issues and applications to retail banking, courts and urban transit. Journal of Productivity Analysis, Dordrecht, v. 4, n. 1/2, , p. 183-210, 1993.

WANG, C. H.;

GOPAL, R. D.;

ZIONTS, S.

Use of data envelopment analysis in assessing information technology impact on firm performance. Annals of Operations Reserch, Dordrecht, n. 73, p. 191-213, 1997.

WARD, P. T. et al.

An analysis of staffing efficiency in U.S. manufacturing: 1983 and 1989. Annals of Operations Research, Dordrecht, n. 73, p. 67-89, 1997.

\section{WEILL, P.}

The relationship between investment in information technology and firm performance in the manufacturing sector. 1989. p. 182. PhD. Dissertation, Stern School of Business, New York University, 1989.

\section{YUE, P.}

Data envelopment analysis and commercial bank performance: a primer with applications to Missouri banks. Federal Reserve Bank of St Louis, St. Louis, v. 74, p. 31-45, jan./feb. 1992. 
